\title{
Report
}

\section{A Study of the Management of Hyponatremia at Mater Dei Hospital, Malta}

\author{
Annalisa Montebello \\ Department of Medicine, Mater Dei Hospital, Msida, Malta \\ Email address: \\ annalisamontebello@gmail.com
}

\section{To cite this article:}

Annalisa Montebello. A Study of the Management of Hyponatremia at Mater Dei Hospital, Malta.Science Journal of Clinical Medicine. Vol. 5, No. 5, 2016, pp. 41-45. doi: 10.11648/j.sjcm.20160505.11

Received: August 11, 2016; Accepted: August 29, 2016; Published: October 15, 2016

\begin{abstract}
Hyponatremia is a frequent electrolyte abnormality in hospital practice. The aim of this study is to assess the prevalence, investigations and outcome of hyponatremia at Mater Dei Hospital (MDH), Malta. All admissions throughout the month of January 2015 were analysed. Patients with low sodium on admission were audited and data collected from iSoft clinical manager and discharge letters to assess if the relevant investigations and treatment changes were performed to correct the hyponatremia. There were 1905 casualty admissions. $16.5 \%$ had hyponatremia on admission. 8.55\% had mild (131-134 mmol/L), $5 \%$ moderate $(125-130 \mathrm{mmol} / \mathrm{L})$ and $2.56 \%$ severe $(<125 \mathrm{mmol} / \mathrm{L})$ hyponatremia. In the severe cohort $69.7 \%$ patients had glucose taken, $57.1 \%$ had thyroid function tests (TFTs), $46.5 \%$ had serum osmolality, $14 \%$ had urine osmolality and electrolytes taken and $18 \%$ had serum cortisol. Rise in sodium in $24 \mathrm{hrs}$ ranged from 1 to $24 \mathrm{mmol}$ with a mean of $8.72 \mathrm{mmol} / \mathrm{L}$. In the moderate cohort $67 \%$ had glucose taken, $45.9 \%$ had TFTs and $43.5 \%$ serum osmolality. Rise in sodium in 24 hrs ranged from 1 to 14 mmol with a mean of $4.7 \mathrm{mmol} / \mathrm{L}$. In the mild cohort $66.7 \%$ had glucose taken, $27.4 \%$ had TFTs and $35.5 \%$ had serum osmolality. Rise in sodium in $24 \mathrm{hrs}$ ranged from 1 to $16 \mathrm{mmol}$ with a mean of $4.1 \mathrm{mmol} / \mathrm{L}$. This study shows the current poor management of severely hyponatremic patients in our medical and surgical wards. There is a definite need to set up local guidelines for the management of such a common electrolyte abnormality.
\end{abstract}

Keywords: Poor Management, Severe, Hyponatremia

\section{Introduction}

Hyponatremia is the commonest electrolyte disturbance seen in hospital practice [1-2]. Mild hyponatremia is usually asymptomatic but if it is severe, with a sodium level of less than $125 \mathrm{mmol} / \mathrm{L}$, it can be a life threatening condition. It can result in seizures and irreversible cerebral oedema [3].

Hyponatremia should be carefully investigated and treated. Serum osmolality, urine osmolality, urine electrolytes and plasma glucose are essential investigations in helping to classify and find out the cause of the hyponatremia. Thyroid function tests, serum cortisol and synachten tests should be done if syndrome of inappropriate ADH secretion (SIADH) is suspected [4].

The treatment of hyponatremia should be carefully managed due to the risk of central pontine myelinolysis if sodium correction is too fast. The rate of sodium correction should be no more than $10-12 \mathrm{mmol} / \mathrm{L}$ in the first 24 hours and $8 \mathrm{mmol} / \mathrm{L}$ in the subsequent 24 hours [4].

\section{Aim}

To assess the prevalence, investigation and clinical outcome of mild, moderate and severe hyponatremia by retrospective analysis in a hospital population.

\section{Methods}

All medical and surgical admissions throughout the month of January 2015 were analysed and their admitting sodium was noted. The patients with hyponatremia on admission were audited and data collected from iSoft clinical manager and 
discharge letters to assess if the relevant investigations and treatment changes were done to correct the electrolyte abnormality.

\section{Results}

There were 1905 casualty admissions throughout the month of January 2015. Mean age of patients was 64.2 years. $52.6 \%$ (1003) were males and 47.3\% (902) were females.

$16.2 \%$ (308) patients had hyponatremia on admission. In this cohort of patients $53.2 \%$ (164) had mild hyponatremia, (Na level: $131-134 \quad \mathrm{mmol} / \mathrm{L}) \quad 30.8 \% \quad(95)$ had moderate hyponatremia (Na level: 125-130mmol/L) 15.9\% (49) had severe hyponatremia (Na level: $<125 \mathrm{mmol} / \mathrm{L}$ ). (Figures 1and 2)

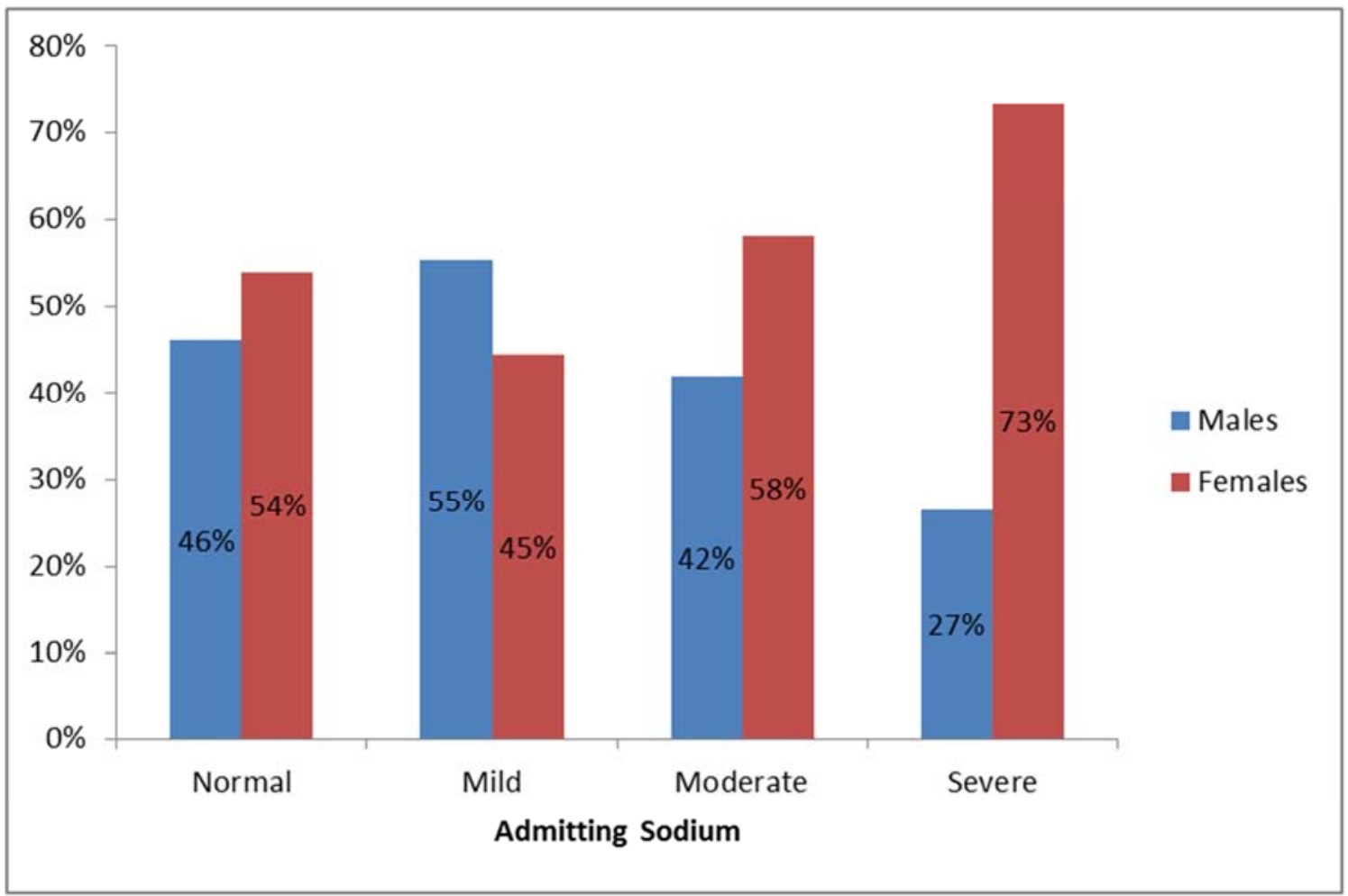

Figure 1. Sodium level on admission.

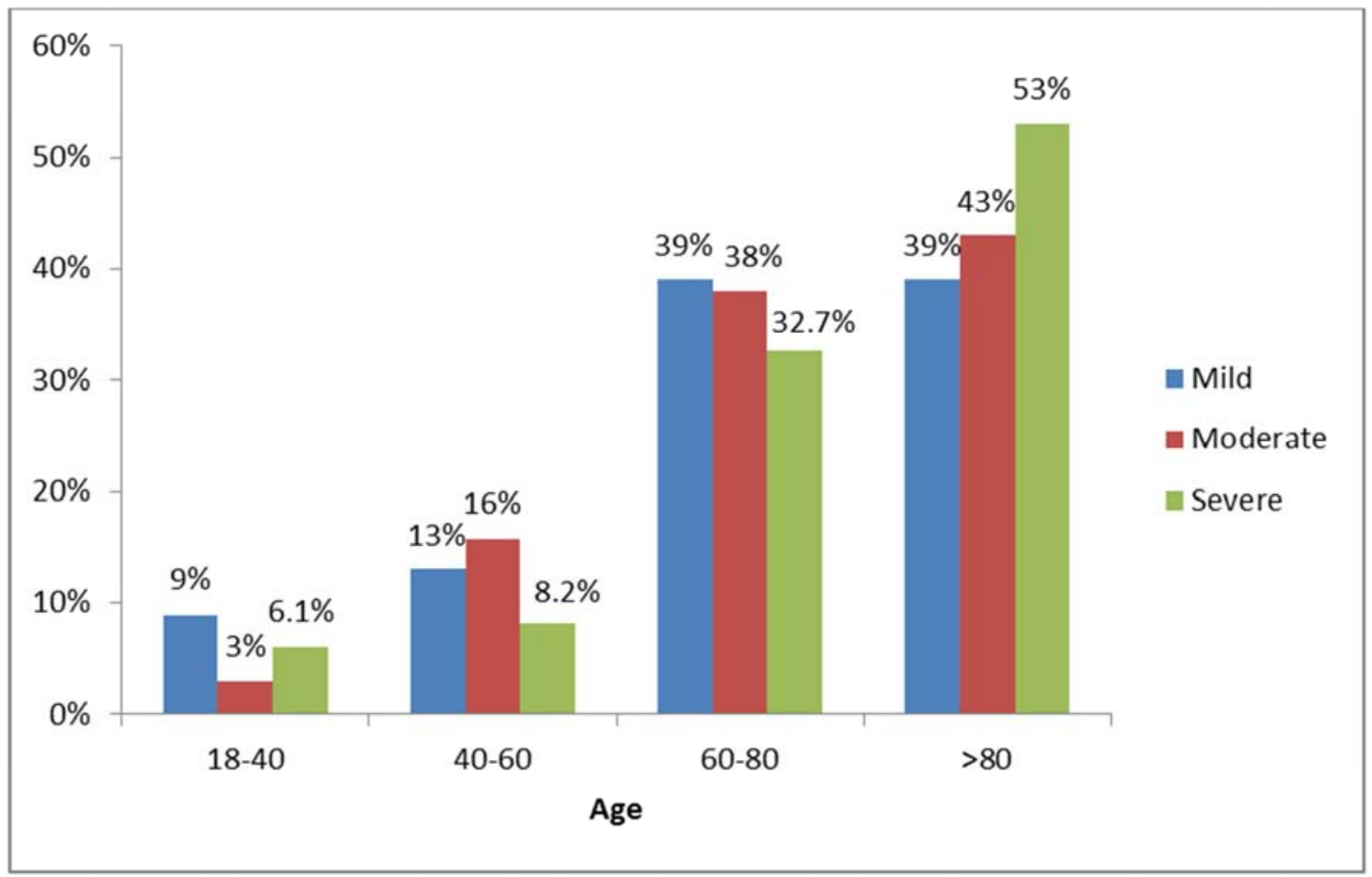

Figure 2. Admissiting Sodium vs. Age. 


\subsection{Severe Hyponatremia}

49 patients admitted with severe hyponatremia were audited. There were $12.2 \%$ (6) patients who died during admission and these were not included in the study. Out of the 43 patients audited $69.7 \%$ (30) were female and 30.2\% (13) were male. The age varied between 21 and 97 with a mean of 77.2 years. Sodium on admission ranged from $112 \mathrm{mmol} / \mathrm{L}$ to $124 \mathrm{mmol} / \mathrm{L}$ with a mean of 119 . Sodium on discharge ranged from $120 \mathrm{mmol} / \mathrm{L}$ to $142 \mathrm{mmol} / \mathrm{L}$ with a mean of 134.5 $\mathrm{mmol} / \mathrm{L}$.

There were $86 \%$ (37) medical admissions and 14\% (6) surgical admissions with severe hyponatremia. The most common causes for admission were congestive heart failure exacerbations at $19 \%$ (8) and pneumonia at $11.9 \%$ (5). Other causes for admission included $4.6 \%$ (2) with diabetic ketoacidosis (DKA), 4.6\% (2) with urosepsis and 4.6\% (2) with transient ischaemic attacks (TIAs). (Figure 3)

$69.7 \%$ (30) patients had their glucose taken whilst $30.2 \%$ (13) patients did not. The glucose result ranged from 5.13 to 47.6 with an average of 11.2 and $80 \%$ of glucose levels being equal to or less than 12.2. $41.8 \%$ (18) patients had TFTs taken whilst 58.1\% (25) did not. 46.5\% (20) had serum osmolality taken whilst $53.4 \%$ (23) did not. Serum osmolality ranged from 249 to 321 with $80 \%$ of patients having a serum osmolality less than or equal to $279 \mathrm{mmol} / \mathrm{L}$. The reference range for serum osmolality at MDH lab is 282 - 300mmol/L. 14\% (6) had urine osmolality and electrolytes taken whilst $86 \%$ (37) did not. Urine $\mathrm{Na}$ ranged from 31 to $125, \mathrm{~K}$ ranged from 4.9 to $57.5, \mathrm{Cl}$ ranged from 22.5 to 144.1 Serum Cortisol was taken in $18.6 \%$ (8) patients whilst $81.4 \%$ (35) did not have a serum cortisol taken. The results for serum cortisol taken ranged from 267 to 1435 . Synachten tests were not taken in 100\%. (Figure 4)

Rise in sodium in $24 \mathrm{hrs}$ ranged from 1 to $24 \mathrm{mmol}$ with a mean of $8.72 \mathrm{mmol} / \mathrm{L}$. Percentage rise in sodium ranged from $0.83 \%$ to $18.3 \%$. $20.9 \%$ (9) had rapid over correction of sodium - more than $10 \mathrm{mmol} / \mathrm{L}$ per 24 hours. A further decrease in serum sodium was noted in two cases $(4.7 \%)$. In these cases the serum sodium further decreased by $2 \mathrm{mmol} / \mathrm{L}$ and $3 \mathrm{mmol} / \mathrm{L}$ respectively.

$47 \%$ (20) were still on angiotensin converting enzyme inhibitors(ACE-I) or angiotensin receptor blockers (ARBs) on discharge, $40 \%$ (17) were still on diuretics, 30\% (13) were still on proton pump inhibitors (PPIs), 21\% (9) were on amlodipine, $4.7 \%$ (2) were on tricyclic anti depressants (TCAs), 2.3\% (1) was on selective seorotonin reuptake inhibitors (SSRIs.)

\subsection{Moderate}

95 patients admitted with moderate hyponatremia were audited. There were $10.5 \%$ (10) patients who died during admission and these were not included in the study. Out of the 85 patients audited 59\% (50) were female and 41\% (35) were male. The age varied between 27 and 94 with a mean of 73.5 years. Sodium on admission ranged from $125 \mathrm{mmol} / \mathrm{L}$ to $134 \mathrm{mmol} / \mathrm{L}$ with a mean of 127.9 . Sodium on discharge ranged from $124 \mathrm{mmol} / \mathrm{L}$ to $148 \mathrm{mmol} / \mathrm{L}$ with a mean of 135.2 $\mathrm{mmol} / \mathrm{L}$.

There were $77.6 \%$ (66) medical admissions and $22.3 \%$ (19) surgical admissions with severe hyponatremia. The most common causes for admission were pneumonia and abdominal pain at $16.4 \%$ (14) patients each. This was followed by congestive heart failure at $9.4 \%$ (8) patients, chest pain, fever and urosepis at 5.8\% (5) patients each and DVT at $3.5 \%$ (3) patients. (Figure 3)

$67 \%$ (57) patients had their glucose taken whilst 32.9\% (28) patients did not. The glucose result ranged from 2.8 to 30 with an average of $8.78 .45 .9 \%$ (39) patients had thyroid function tests - TFTs taken whilst 54\% (46) did not have TFTs taken. $43.5 \%$ (37) had serum osmolality taken whilst $56.4 \%$ (48) did not. Serum osmolality ranged from 250 to $331 \mathrm{mmol} / \mathrm{L}$. The reference range for serum osmolality at $\mathrm{MDH}$ lab is 282 $300 \mathrm{mmol} / \mathrm{L}$. 4.7\% (4) had urine osmolality and electrolytes taken whilst $94.3 \%$ (81) did not. Urine Na ranged from 22 to $159, \mathrm{~K}$ ranged from 4.9 to $29.9, \mathrm{Cl}$ ranged from 17.8 to 113.Serum Cortisol was not taken in any of the patients. (Figure 4)

Rise in sodium in $24 \mathrm{hrs}$ ranged from 1 to $14 \mathrm{mmol}$ with a mean of $4.7 \mathrm{mmol} / \mathrm{L}$. \% rise in sodium ranged from $0 \%$ to $10 . \%$ with an average of $4 \%$.A further decrease in serum sodium was noted in six cases $(7 \%)$. In these cases the serum sodium further decreased by $1 \mathrm{mmol} / \mathrm{L}$ to $3 \mathrm{mmol} / \mathrm{L}$.

$31.7 \%$ (27) were still on ACE/ARBs on discharge, $55.2 \%$ (47) were still on diuretics, $38.8 \%$ (33) were still on PPIs, $10.6 \%$ (9) were on amlodipine, $1.1 \%$ (1) was on TCAs, and $8.2 \%$ (7) was on SSRIs.

\subsection{Mild}

164 patients admitted with moderate hyponatremia were audited. There were $17.7 \%$ (29) patients who died during admission and these were not included in the study. Out of the 135 patients audited $43.7 \%$ (59) were female and 56.2\% (76) were male. The age varied between 22 and 97 with a mean of 71 years. Sodium on admission ranged from $130 \mathrm{mmol} / \mathrm{L}$ to $134 \mathrm{mmol} / \mathrm{L}$ with a mean of 132.5 Sodium on discharge ranged from $128 \mathrm{mmol} / \mathrm{L}$ to $149 \mathrm{mmol} / \mathrm{L}$ with a mean of 137.4 $\mathrm{mmol} / \mathrm{L}$.

There were $78.5 \%$ (106) medical admissions and 21.5\% (29) surgical admissions with severe hyponatremia. The most common causes for admission were 14.8\% (20) abdominal pain , $11.9 \%$ (16) presyncope , $11.1 \%$ (15) pneumonia and $8.8 \%$ (12) CHF, $8.1 \%$ (11) chest pain. (Figure 3)

$66.7 \%$ (90) patients had their glucose taken whilst $54.8 \%$ (74) patients did not. The glucose result ranged from 1.5 to 31.8 with an average of $10.75 .27 .4 \%$ (37) patients had thyroid function tests - TFTs taken whilst $72.6 \%$ (98) did not have TFTs taken. 35.5\% (48) had serum osmolality taken whilst $64.4 \%$ (87) did not. Serum osmolality ranged from 283 to $327 \mathrm{mmol} / \mathrm{L}$. The reference range for serum osmolality at MDH lab is $282-300 \mathrm{mmol} / \mathrm{L}$. None had urine osmolality taken. Serum Cortisol was taken in one patient. (Figure 4) 
Rise in sodium in $24 \mathrm{hrs}$ ranged from 1 to $16 \mathrm{mmol}$ with a further decreased by 1 to $8 \mathrm{mmol} / \mathrm{L}$.

mean of $4.1 \mathrm{mmol} / \mathrm{L}$. \% rise in sodium ranged from $0 \%$ to $10.8 \% \quad 37.7 \%$ (51) were still on ACE/ARBs on discharge, $41.5 \%$ with an average of $3.6 \%$. A further decrease in serum sodium $\quad(56)$ were still on diuretics, $28 \%$ (38) were still on PPIs, 9.6\% was noted in eight cases. In these cases the serum sodium (13) were on amlodipine, 8.14\% (11) was on SSRIs.

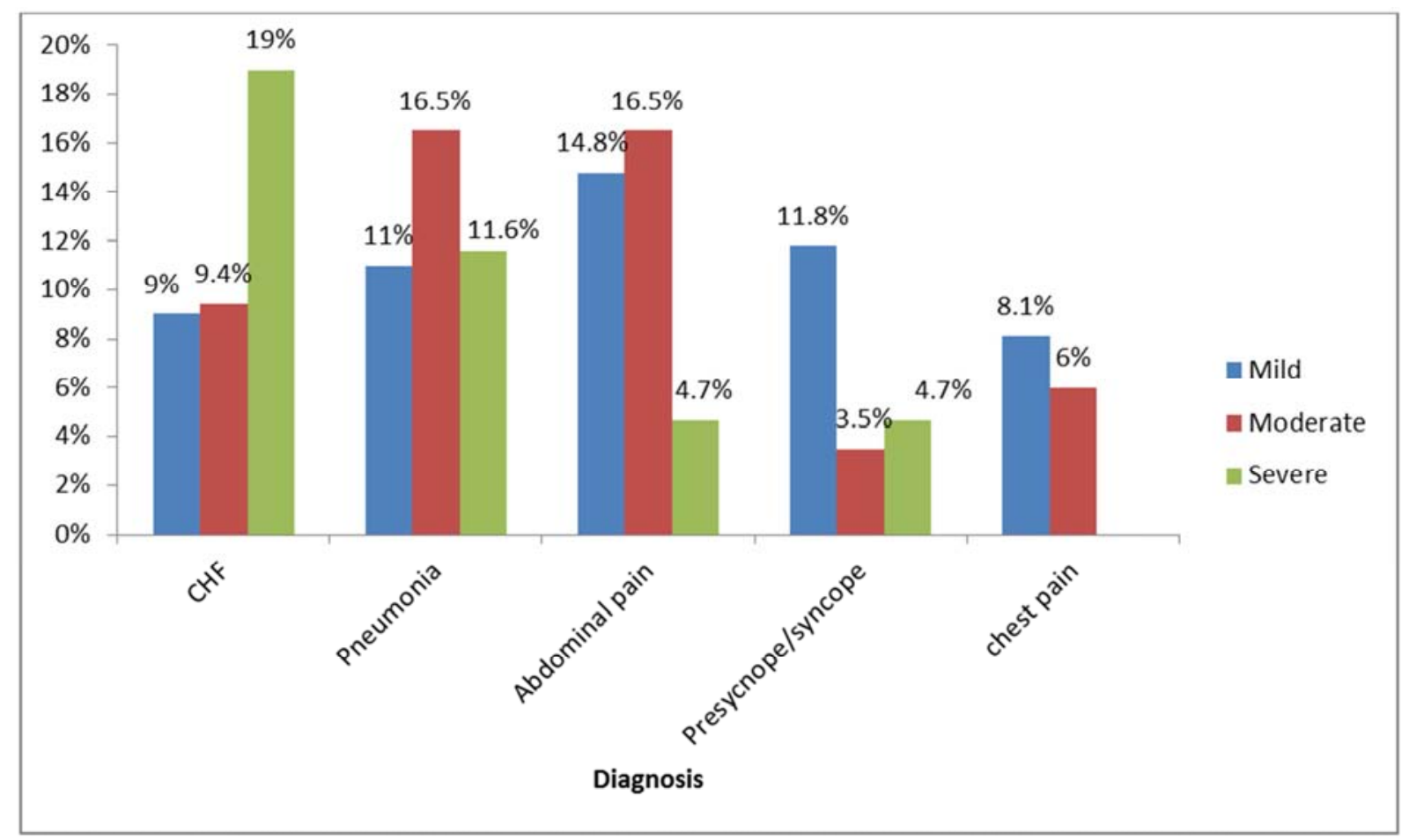

Figure 3. Admitting Sodium vs. Diagnosis.

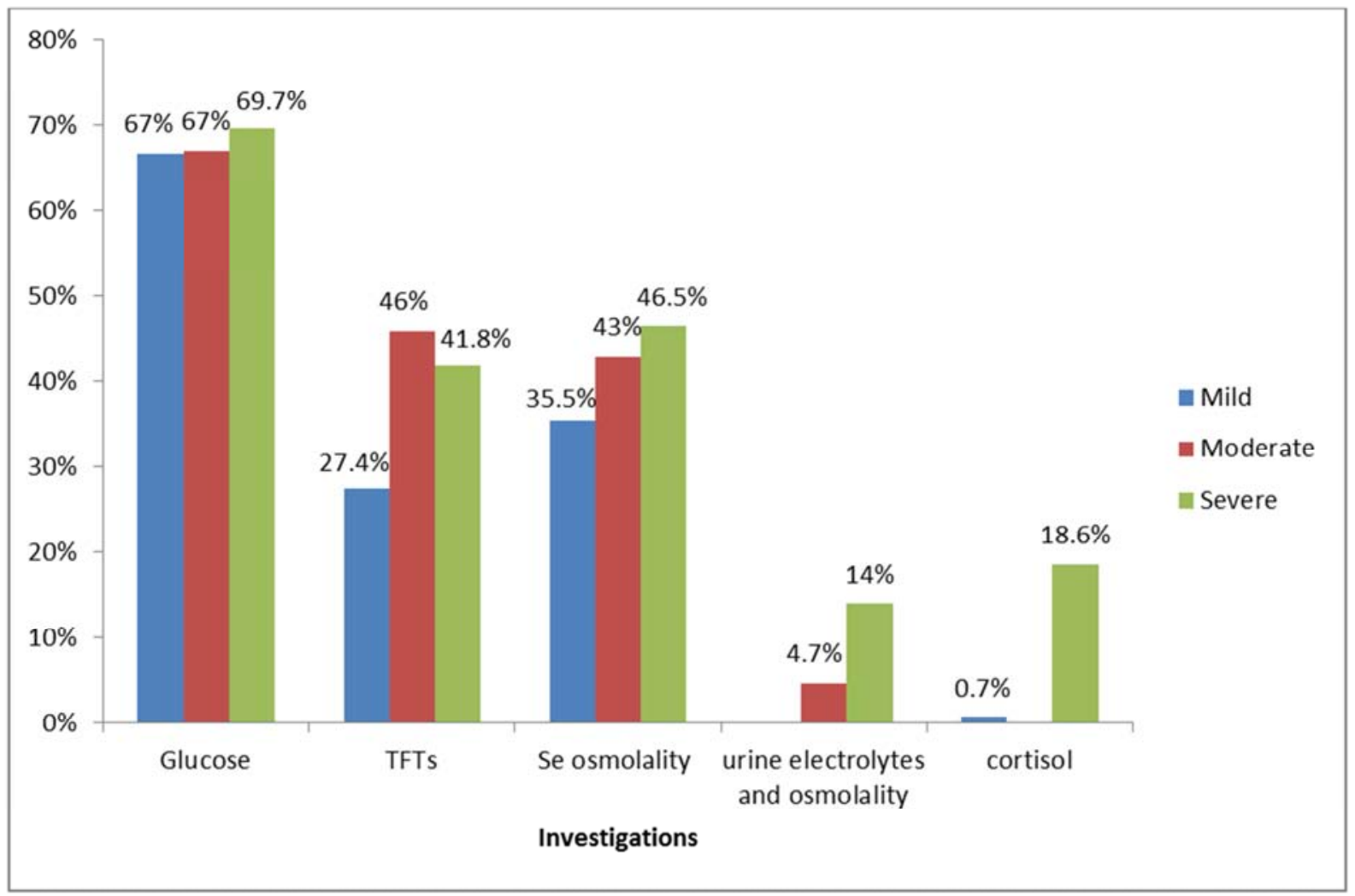

Figure 4. Investigations performed in all classes of Hyponatremia. 


\section{Discussion}

This study shows how frequent hyponatremia is in our day to day hospital practice. It is usually a complication of other medical illnesses; most frequently heart failure, pneumonia, renal failure and liver failure [5]. Mild hyponatremia is the commonest abnormality. The majority of patients with severe hyponatremia are admitted under medical firms. Diuretics were found to be the major cause of hyponatremia as congestive heart failure was the most common reason for admission at in both the moderate and the severe hyponatremia groups.

We are still lacking behind in the correct investigations for hyponatremia. The majority of patients in all the classes of hyponatremia did not have serum osmolality, urine osmolality or urine electrolytes taken. These are the three most basic investigations necessary to classify hyponatremia into hypotonic, isotonic or hypertonic volume status [5]. Following this classification we can administer the appropriate treatment. Hypotonic hyponatremia can be treated with fluids. Hypertonic hyponatremia treatment depends on the underlying disorder [5]. Serum glucose is also an important investigation. In hyperglycemic patients the sodium concentration should be corrected for the effect of glucose to exclude hypertonic hyponatremia [6]. Acute hyponatremia ( $<48$ hours) can be corrected more quickly than chronic hyponatremia.

In the severe hyonatremia cohort $20.9 \%$ had too rapid correction of sodium which can result in central pontine myelinolysis. Central pontine myelinolysis is a non inflammatory demyelization of the brain that occurs when there is too rapid correction of hyponatremia. $51.1 \%$ (22) patients in the severe hyponatremia class were still hyponatremic $(<135 \mathrm{mmol} / \mathrm{L})$ on discharge whilst $37.6 \%$ (32) in the moderate and $25.9 \%$ (35) in the mild were also hyponatremic on discharge. This shows we are currently under treating all classes of hyponatremia and patients are being discharged with a low sodium level.

Of note, the majority of patients in all classes of hyponatremia were still on sodium lowering drugs on discharge. ACE inhibitors and diuretics were the most common drugs associated with hyponatremia in all the classes. Other common drugs causing hyponatremia include PPIs, amlodipine, TCAs and SSRIs.

\section{Conclusion}

This study clearly shows the current poor management of hyponatremic patients in our medical and surgical wards. One limitation in this study was that the medical notes were not reviewed in this audit to establish if the volume status was documented, to investigate inpatient management and to assess why sodium lowering drugs could not be stopped. There is a definite need to set up local guidelines for the management of hyponatremia in the local wards.

\section{References}

[1] Baran D, Hutchinson T. The outcome of hyponatremia in a general hospital population. Clin Nephrol. 1984; 22(2): 72-6.

[2] Gill G, Leese G. Hyponatraemia: biochemical and clinical perspectives. Postgraduate Medical Journal. 1998; 74(875): 516-523.

[3] Siddique H, Kaja R, Daggett P. An audit on the management of severe hyponatremia in a hospital population. Presented at 197th Meeting of the Society for Endocrinology 2006, London, UK Endocrine Abstracts. 2006; 12: P9.

[4] Spasovski G, Vanholder R, Allolio B, Annane D, Ball S, Bichet $\mathrm{D}$ et al. Clinical practice guideline on diagnosis and treatment of hyponatraemia. European Journal of Endocrinology. 2014;171(1):X1-X1.

[5] Simon E, Mehrdad Hamrahian S, Teran F. Hyponatremia: Practice Essentials, Pathophysiology, Epidemiology [Internet]. Emedicine.medscape.com. 2016 [cited 28 August 2016]. Available from:

http://emedicine.medscape.com/article/242166-overview

[6] Sterns R. Diagnostic evaluation of adults with hyponatremia [Internet]. Uptodate.com. 2016 [cited 28 August 2016]. Available from:

http://www.uptodate.com/contents/diagnostic-evaluation-of-ad ults-with-hyponatremia

[7] Ghaffar I, Downie P, Ahmad B, Thorogood N, Thomas P, Bradley K. Hyponatremia: an audit of the initial investigation and management. Endocrine Abstracts. 2015;

[8] Saeed B. Severe hyponatraemia: investigation and management in a district general hospital. Journal of Clinical Pathology. 2002; 55(12): 893-896.

[9] Clayton J. Severe hyponatraemia in medical in-patients: aetiology, assessment and outcome. QJM. 2006; 99(8): 505-511.

[10] Reynolds RM, Padfield PL \& Seckl JR. Disorders of sodium balance. BMJ 2006332 (7543) 702-705.

[11] Reynolds RM \& Seckl JR. Hyponatraemia for the clinical endocrinologist. Clin Endocrinol (Oxf) 200563 (4) 366-374. 